\title{
O USO DE ENERGIA DE MICROONDAS PARA POLIMERIZAÇÃO DE RESINAS ACRÍLICAS
}

\author{
The use of microwave energy to polymerize acrylic resin
}

\author{
Sávio Marcelo Leite MOREIRA-DA-SILVA ${ }^{1}$; Márcio José \\ Fraxino BINDO'; Moira Pedroso LEÃO ${ }^{3}$
}

\begin{abstract}
RESUMO
Este trabalho apresenta uma ampla revisão da literatura sobre polimerização de resinas acrílicas por microondas, estabelecendo uma comparação entre este método e o tradicional em água aquecida, e sua influência nas propriedades físicas, mecânicas e biológicas das resinas.
\end{abstract}

Palavras-chave: Resinas acrílicas; Microondas; Dentaduras; Bases para dentadura.

\begin{abstract}
This paper presents an ample literature revision about acrylic resins polymerization by microwaves energy, establishing a comparison between this method and the traditional water bath, and its influences in the mechanic, biologic and physic resins properties.
\end{abstract}

Keywords: Acrylic resins; Microwaves; Dentures; Denture base

\footnotetext{
${ }^{1}$ Mestre em Reabilitação Oral pela Faculdade de Odontologia de Bauru - USP. Professor de Prótese Dentária no nível profissional, graduação e pós-graduação da Universidade Federal do Paraná e do Centro Universitário Positivo - UnicenP.

${ }^{2}$ Doutor em Prótese Dentária pela Faculdade de Odontologia da Universidade de São Paulo - USP. Professor de Prótese Dentária na graduação e pós-graduação da Universidade Federal do Paraná e do Centro Universitário Positivo - UnicenP.

${ }^{3}$ Mestre em Implantodontia pela Curso de Odontologia da Universidade Federal de Santa Catarina - UFSC. Professor de Prótese Dentária na graduação e pós-graduação na Universidade Federal do Paraná e do Centro Universitário Positivo - UnicenP.
} 


\section{INTRODUÇÃO}

As resinas acrílicas começaram a ser utilizadas em odontologia a partir dos anos 30 como material substitutivo à borracha vulcanizada na confecção de bases de próteses totais. Suas características atendem aos preceitos estéticos e funcionais, contudo não são totalmente ideais. ${ }^{1} \mathrm{Na}$ busca por aperfeiçoamento destas características, várias pesquisas, desde então, têm sido desenvolvidas. Em 1968, NISHII ${ }^{2}$ experimentou um novo método de polimerização para as resinas termopolimenrizáveis. Ao invés do aquecimento em banho de água, onde a difusão do calor se faz vagarosamente do exterior para o centro da mufla, foi utilizada energia de microondas como método de aquecimento, reduzindo o tempo de polimerização de 2 horas e 30 minutos para apenas 9 minutos, além de facilitar o processamento. $^{2}$

Este método, chamado dielétrico, consiste na emissão de ondas eletromagnéticas capazes de atravessar materiais refratários e promover vibração de elétrons na molécula de monômero. Esta vibração promove o aumento de temperatura da massa ativando a reação de polimerização. O estudo de NISHII ${ }^{2}$ (1968) apontava a necessidade de aperfeiçoamento da técnica e do material usado para polimerização. Fato que gerou uma série de estudos posteriores.

O objetivo deste trabalho é mostrar uma comparação entre as técnicas de polimerização convencional por aquecimento em banho de água e a técnica de microondas, mostrando suas vantagens, desvantagens e interferências nas propriedades das resinas acrílicas.

\section{POLIMERIZAÇÃO DAS RESINAS ACRÍLICAS}

\section{Química das resinas}

As resinas acrílicas para base de dentadura são compostos obtidos a partir da polimerização do metilmetacrilato. São apresentadas comercialmente em dois frascos, um contendo pó formado por micropérolas de polímero (polimetilmetacrilato), e no outro o monômero (metilmetacrilato) que é um solvente orgânico que em temperatura ambiente apresenta-se em forma líquida. Normalmente o líquido está adicionado de uma pequena quantidade hidroquinona (cerca de 0,2\%) que serve para inibir uma indesejável polimerização do monômero.

Este monômero quando polimerizadas formam uma cadeia linear na qual as moléculas estão ligadas covalentemente, formando o polimetilmetacrilato. ${ }^{3}$ Este processo de polimerização necessita ser iniciado por uma molécula capaz de se tornar energizada e energizar as moléculas de monômero através da liberação sucessiva de radicais. Como o metilmetacrilato tem dificuldades para iniciar a reação, uma proporção de peróxido de benzoíla é adicionada ao polímero para cumprir este papel.

PHILLIPS $^{3}$ (1993) descreve os estágios da polimerização como indução, propagação e terminação. A indução é o estágio no qual a molécula de iniciador torna-se energizada e passa a transferir sua energia para as moléculas do monômero. O peróxido de benzoíla é o iniciador mais utilizado na polimerização das resinas acrílicas. Sua ativação é feita pela temperatura, quando acima de $55^{\circ} \mathrm{C}$, ou pela reação com uma amina terciária, é o caso das resinas autopolimerizáveis que contém na composição do líquido a dimetil-p-toluidina. 
Outros sistemas podem promover a ativação do peróxido de benzoíla, é o caso da canforoquinona com aminas, capazes de sofrer ação da luz visível tornando a resina fotoativada. Outra fase seqüente da polimerização é a propagação, as moléculas de monômero se juntam sucessivamente à cadeia. A terminação representa o encerramento da cadeia por ligação direta entre cadeias poliméricas ou por adição de hidrogênio de uma cadeia em crescimento. $O$ processo pelo qual uma cadeia é incorporada a outra gerando um novo núcleo reativo é denominado de transferência de cadeia.

No processo de polimerização a conversão de monômero em polímero não é total, gerando um percentual de monômero residual que varia para mais ou menos devido à interferências de vários fatores durante o processamento.

\section{Polimerização por Microondas}

O forno de microondas é um aparelho capaz de emitir ondas eletromagnéticas a uma freqüência de $2450 \mathrm{MHz}$, a partir de um magnetron, aparelho a vácuo que transforma energia elétrica alternada em microonda. ${ }^{27} \mathrm{~A}$ onda emitida é capaz de atravessar objetos sólidos até atingir uma molécula que a absorva e consequentemente vibre. É o caso do metilmetacrilato, seus elétrons passam a ter maior velocidade aumentando consequentemente a temperatura. Esta elevação de temperatura é irradiada para a massa e promove a indução da polimerização.

Este processo difere-se do método convencional de aquecimento por água justamente porque o aquecimento pode ocorrer simultaneamente em todos os pontos da massa de resina, enquanto o aquecimento no método de indução de temperatura necessita de bons condutores para se propagar.
A energia de microondas pode ser utilizada para redução do tempo de formação da fase plástica e produzindo bons resultados inclusive na homogeneização da massa. ${ }^{4}$

Tecnicamente, o processo de inclusão e prensagem da base da dentadura para polimerização por microondas é a mesma do método convencional. A diferença está na mufla, que deve ser plástica, normalmente reforçada com fibras de vidro, uma vez que as metálicas refletem as microondas, impedindo-as de penetrar no interior do aparelho. As muflas plásticas, idealizadas e utilizadas pela primeira vez por KIMURA, et al. ${ }^{5}$ (1984) têm desvantagens em relação as metálicas, pois expandem, têm vida útil menor, uma vez que fraturam após vários processamentos, e limitam a pressão de prensagem a 1200 psi. $^{6}$

Depois do processo de inclusão o molde deve ser levado ao forno de microondas a uma potência de $500 \mathrm{~W}$ por 3 minutos em forno de microondas convencional. ${ }^{4,}$, , 8, 9, 10, 11, 12

O prato giratório proporciona uma maior e mais homogênea penetração de ondas na mufla, uma vez que a conduz por diversas posições no campo eletromagnético.

O comportamento da temperatura das resinas no interior da mufla é diferente para os métodos de aquecimento. A temperatura da resina e do gesso de revestimento, em muflas plásticas submetidas ao campo de microondas, eleva-se de maneira muito rápida e gradual. É necessário apenas 1 minuto e meio para a massa atingir $65^{\circ} \mathrm{C}$, e sua temperatura máxima não ultrapassa $120^{\circ} \mathrm{C} .^{5}$ A diferença fundamental do aquecimento em banho de água é o pico de aquecimento exotérmico desencadeado quando a massa atinge cerca de $65^{\circ} \mathrm{C}$, podendo causar porosidade por evaporação de monômero. 


\section{INTERFERÊNCIA DO MÉTODO DE POLIMERIZAÇÃONAS PROPRIEDADESDASRESINAS}

\section{Porosidade}

A porosidade é um fenômeno multifatorial ${ }^{13}$ e a temperatura de polimerização é um fator preponderante na determinação de sua magnitude.

Os poros podem ser internos, gerando uma área de fragilidade, e como conseqüência uma maior vulnerabilidade à fratura. Quando são superficiais, dificultam a limpeza das mesmas podendo provocar até mesmo estomatites protéticas. ${ }^{14}$

A espessura exerce papel preponderante na formação de bolhas internas. ${ }^{12}$ Apesar de a literatura ter constantemente reafirmado que não há diferença de porosidade entre as técnicas de cura por microondas ou banho de água para corpos finos (menor que $3 \mathrm{~mm}$ ), , ${ }^{15,16}$ se aumentarmos a espessura para cerca de $1 \mathrm{~cm}$, uma maior porosidade pode aparecer nas resinas quando polimerizadas por microondas. ${ }^{16}$ Nestes casos, a diminuição da potência com aumento do tempo de polimerização pode ser eficiente na redução da porosidade. ${ }^{15,16}$

O estudo de YANNIKAKIS, et al. ${ }^{17}$ (2002) mostra que a porosidade pode ser aumentada pela polimerização em microondas mesmo em corpos finos, comparada ao banho de água no ciclo de 2 horas. Afirma ainda que a diminuição da potência com aumento do tempo de radiação pode ajudar a reduzir porosidade, até mesmo em resinas próprias para microondas em que o fabricante preconiza um ciclo curto com potência elevada.

A temperatura de ativação do peróxido de benzoíla pode desencadear um processo de auto-aquecimento exotérmico da resina, cuja intensidade é proporcional a velocidade de elevação da temperatura. Tal fenômeno é descrito por PHILLIPS ${ }^{18}$ (1993) através de um gráfico comparando a temperatura da água, do gesso e da resina num método de termopolimerização convencional. Este superaquecimento seria o principal fator provocante de porosidade interna.

As resinas convencionais, quando polimerizadas por microondas, apresentam maior porosidade que as desenvolvidas propriamente para esta técnica. ${ }^{8}$ As resinas próprias para microondas são desenvolvidas com um monômero obtido da mistura de etil e metilmetacrilato, ou pode conter tetra ou trietilenoglicol que são substâncias que possuem baixa pressão de vapor em temperaturas entre 100 e $150^{\circ} \mathrm{C}$, justificando um menor aparecimento de porosidade neste tipo de resina. ${ }^{11,13}$

O resfriamento tem mostrado interferência no aparecimento de porosidade. A porosidade é maior em resinas resfriadas subitamente, comparadas àquelas esfriadas em temperatura ambiente. ${ }^{15}$ Entretanto, SANDERS, et al. ${ }^{13}$ (1987) encontraram menor porosidade em dois dos quatro tipos de resina testadas pelo resfriamento rápido (45 minutos em água gelada), após polimerização por microondas, comparando-as com resinas resfriadas em câmara de resfriamento em 2 horas e meia.

\section{Dureza}

Parece haver consenso entre vários autores estudados de que não existe diferença significante nas diversas medidas de dureza das resinas acrílicas se submetidas ao método de polimerização por microondas ou por banho de água. ${ }^{6,16,19,20}$

\section{Resistência ao impacto}

BLAGOGEVIC e MURPHY'19 (1999) mostraram em seu trabalho que o método de 
polimerização interfere na resistência ao impacto das resinas acrílicas. Comparando polimerização por microondas a 600W por 3 minutos com banho de água a $70^{\circ} \mathrm{C}$ por 14 horas, seguido de 3 horas em ebulição, suas conclusões apontaram uma maior resistência para as resinas curadas pelo segundo método. MEMON, et al. ${ }^{21}$ (2001) confirmaram este resultado, afirmando, contudo, que esta diferença é pequena.

\section{Absorção de água}

A absorção de água é um fenômeno que atinge as resinas nos três primeiros meses após a confecção da prótese. Esse ganho de água altera dimensionalmente a resina, provocando expansão. ${ }^{10,14}$ Proporcionalmente, as resinas polimerizadas por microondas, quando comparadas às polimerizadas por banho de água, mostram maior absorção. ${ }^{12}$

SADAMORI, et al. ${ }^{12}$ (1997) mostram em seu estudo que quanto maior a espessura da resina maior é a absorção de água, e que o método de polimerização interfere na capacidade de absorção de água. Já o trabalho de BRAUN et al. ${ }^{14}$ (1995) não mostra diferença estatisticamente significante entre polimerização em água e em microondas, nem entre resinas convencionais e próprias para microondas irradiadas pelo mesmo método.

DEL BEL CURY, et al. ${ }^{10}$ (1994) e BRAUN, et al. ${ }^{14}$ (1995) afirmam que uma menor absorção de água pode estar relacionado com a presença de agentes de ligação na resina, ao contrário do que diz JAGGERS e HUGGET $^{22}$ (1990) que testaram a adição de agente de ligação em resinas para base de dentadura, em proporção de 0\% a 100\%, e não encontraram diferença no coeficiente de difusão de água nos corpos. Entretanto, ambos concordam que a presença de frinchas pode aumentar a capacidade de absorção de água, e agentes de ligação aumentam a resistência à fratura.

\section{Estabilidade de cor}

KIMURA, et al. ${ }^{4}$ (1983) não encontraram alterações de cor significativas em resinas polimerizadas por microondas. MAY, et al. ${ }^{23}$ (1996) testaram várias resinas pelo método de aceleração de tempo, e constatou uma menor alteração de cor em resinas termopolimerizáveis convencionais polimerizadas por microonda, que resinas próprias para microondas, mas não encontrou diferença estatisticamente significante na comparação dos métodos.

\section{Citotoxidade}

Os aspectos clínicos da toxicidade das resinas acrílicas nos tecidos bucais, intimamente contatados pelas bases das dentaduras, são de difícil determinação. Esta análise esta diretamente ligada ao estudo da biocompatibilidade dos materiais dentários.

SHERIDAN, et al. ${ }^{24}$ (2002) avaliaram a citotoxidade das resinas para base de dentadura avaliando a viabilidade celular, dada pela atividade enzimática mitocondrial dos fibroblastos gengivais. Seu trabalho mostrou menor toxicidade de um tipo de resina termocurada em relação à resina própria para microondas.

\section{Liberação de monômero residual}

A concentração de monômero residual é o parâmetro mais importante na determinação de propriedades das resinas, segundo o estudo de JAGGERS ${ }^{25}$ (1978), que mostrou correlação deste item com dureza, resistência à tração e absorção de água.

A liberação de monômero residual não indica exatamente a sua quantidade em um corpo de resina, uma vez que somente o monômero das regiões superficiais seria liberado para o meio. Entretanto, sua liberação pode estar relacionada com a toxicidade da resina, produzindo respostas indesejáveis ao tecido mucoso de suporte das dentaduras. ${ }^{9}$ 
O teor de monômero residual por todo o corpo da resina indica o grau de polimerização final do monômero, ou seja, a conversão de monômero em polímero. ${ }^{9}$ Métodos de cromatografia podem ser usados para determinar quantitativamente este valor. Por este método, BLAGOGEVIC e MURPHY ${ }^{19}$ (1999) mostraram maior concentração em resinas polimerizadas por microondas, e menor nas mesmas quando polimerizadas em banho de água, embora valha a ressalva do uso do ciclo longo (14 horas a $70^{\circ} \mathrm{C}$, seqüenciados por 3 horas em fervura).

Usando outra metodologia de medida, apresentada como muito precisa, BARTOLONI, et al. ${ }^{9}$ (2000) não encontraram diferença no grau de polimerização entre as duas técnicas de polimerização, embora o ciclo de termopolimerização adotado tenha sido de $74^{\circ} \mathrm{C}$ por 9 horas sem terminação em ebulição. Esta terminação, para AL DOORI e HUGGETT ${ }^{15}$ (1988), é fundamental para maior conversão de monômero.

DEL BEL KURY, et al. ${ }^{26}$ (2001) mostraram que a inclusão em mufla utilizando muralha de silicone alimenta a concentração de monômero residual, comparada ao preenchimento total da mufla com gesso. Esta maior concentração não é mais verificada se os espécimes permanecerem em imersão em água por cinco dias.

\section{Adesão de dentes artificiais}

KIMURA, et al. ${ }^{4}$ (1983) não encontraram diferença estatística na adesão de dentes de resina em polímeros curados pelas duas técnicas discutidas. Entretanto, TERAOKA e TAKAHASHI ${ }^{27}$ (2000) estudando um tipo de resina especial para polimerização por microondas, mostraram menor adesão desta, comparada com outra resina termocurada. Este resultado foi ratificado por SCHNEIDER, et al. ${ }^{28}$ (2002) em um estudo com metodologia parecida.

\section{Resistência transversal}

MONTEIRO NETTO, et al. ${ }^{29}$ (1999) não encontraram diferença estatisticamente significante para resina termicamente ativadas em ciclo de polimerização em forno de microondas convencional por 3 minutos a $665 \mathrm{~W}$, comparadas a mesma polimerizada em banho de água por 1 hora e 30 minutos a $70^{\circ} \mathrm{C}$ e elevada a $100^{\circ} \mathrm{C}$ por 1 hora. Tal resultado confirma trabalhos anteriores, que compararam por vários métodos os dois tipos de polimerização. ${ }^{6,26,21}$ SILVA, et al. ${ }^{30}$ (2002) mostraram ainda, que a adição de pigmentos intrínsecos e fibras acrílicas numa proporção de até 1,5\% não altera a resistência transversal de uma resina polimerizada por microondas.

\section{Adaptação ao modelo de trabalho}

A adaptabilidade da base da dentadura é qualitativamente superior àquelas curadas por banho de água. 4, 7, 11 Para tanto, é importante que as microondas aqueçam o molde de gesso ou homogeneízem a fase de massa da resina. ${ }^{4}$ Essa adaptabilidade das resinas de microondas é maior na região de crista que na região do palato, ${ }^{5,31}$ e maior na região anterior que na posterior, com piores resultados na região do post dam. ${ }^{1}$

ALMEIDA, et al. ${ }^{1}$ (1999) não encontraram diferença estatística nas distorções para as duas técnicas. Para BARBOSA, et al. ${ }^{7}$ (1998) os resultados mostrados pela literatura, até então, são inconclusivos para a comparação da influência da técnica de polimerização na determinação do grau de adaptação, devido as diferenças consideráveis entre os desenhos experimentais aplicados por cada autor.

A adição de pó de alumínio, numa concentração superior a $10 \%$, no gesso de revestimento da mufla, cria um maior aquecimento do gesso irradiado por microondas. Se adicionarmos $20 \%$ e $30 \%$, 
respectivamente, no revestimento da mufla e no modelo de gesso incluído, proporcionaremos um maior aquecimento pelo lado tecidual da dentadura, aumentando de $20 \%$ a $30 \%$ a adaptação das bases. ${ }^{32}$

TERAOKA e TAKAHASHI ${ }^{27}$ (2002), na tentativa de desenvolver uma técnica para a obtenção de dentaduras com máxima adaptação ao modelo de gesso, e por conseqüência, na mucosa de suporte, descreveram cinco características que devem nortear o processo de polimerização:

- menor quantidade de água livre no gesso que reveste a mufla;

- a resina em forma plástica deve ser injetada rapidamente no interior da mufla e mantida numa pressão abaixo de 3,5 $\mathrm{Mpa}$, até a polimerização ser completada;

- usar um mínimo necessário de calor para a completa polimerização da resina;

- iniciar a polimerização uniformemente, de maneira que a superfície que contata a mucosa polimerize-se primeiro e continue em direção aos dentes de estoque e a resina do conduto de injeção, na porção superior do molde, seja a ultima a se polimerizar;

- iniciar a polimerização imediatamente após a resina ser injetada na mufla.

\section{Alteração dimensional linear}

É preciso considerar que a polimerização das resinas acrílicas provoca uma contração linear de $0,2 \%$ a $0,7 \%{ }^{18}$

Quanto maior a espessura da base da resina maior será a alteração dimensional linear. Entretanto, os métodos de polimerização por microondas e banho de água não mostram diferença quando submetemos as resinas a imersão em água destilada, por 90 dias, após a polimerização. ${ }^{12}$
Comparando resinas termopolimerizadas em banho de água, em ciclo de 90 minutos a $70^{\circ} \mathrm{C}$ seguindo 30 minutos a $100^{\circ} \mathrm{C}$, com resinas para microondas, polimerizadas em ciclo de 3 minutos a 500W em forno de microondas convencional, SADAMORI, et al. ${ }^{12}$ (1997) observaram que, depois de removidos das muflas, os corpos de prova polimerizados em água quente contraíam em menor proporção aos polimerizados por microondas. Entretanto, submergindo os corpos em água destilada observou-se que, nas primeiras 24 horas ambas as resinas continuavam a contrair levemente. Em seguida, iniciavam um processo de expansão por 90 dias, de tal forma que as resinas de microondas, expandindo mais, alcançavam dimensões, não diferente significativamente, das resinas convencionais.

\section{OUTRAS TÉCNICAS DE APLICAÇÃO DAS RESINAS ACRÍLICAS}

\section{Reembasamento}

SADAMORI, et al. ${ }^{33}$ (1995) testaram vários tipos de resina em reembasamento de próteses totais. Dentre as resinas auto, termo, foto e polimerizadas por microondas, as resinas termo apresentaram melhor adaptação, significativamente melhor que as resinas de microondas. Todas as resinas tiveram melhor adaptação no fundo de vestíbulo e pior adaptação no palato, exceto a resina de microondas, comportando-se exatamente da maneira oposta.

Bases de prótese, construídas de resina especial para microondas em um primeiro ciclo, e submetidas a um segundo ciclo de polimerização para reembasamento, apresentam maiores distorções que bases convencionais termocuradas submetidas a mesma condição. ${ }^{11}$ GARCIA e DEL BEL CURY $^{11}$ (1996) explicam tal fato afirmando que a temperatura de transição vítrea das resinas termopolimerizadas convencionais é maior. Por isso as resinas de microondas facilmente 
ultrapassariam esta temperatura num segundo ciclo de irradiação, sofrendo indução de novas tensões dada pela diferença dos coeficientes de contração da resina e do gesso. Neste estudo os autores concluíram que o reembasamento com resina de microondas adapta-se melhor ao modelo padrão que aquele com resinas normais, desde que a base da prótese tenha sido construída inicialmente por resinas convencionais termopolimerizadas. Quanto a porosidade, não houve diferença significante entre antes e depois do reembasamento.

\section{Polimerização de resina em contato com metais}

A energia de microondas pode ser utilizada para polimerização de resinas acrílicas contendo no seu interior uma sela metálica, apresentando resultados adequados para dureza superficial e menores conteúdos de monômero residual. ${ }^{20}$ Fato importante para a estilização deste material na construção de próteses parciais removíveis. O metal contido no interior da resina não contribui para o aumento da porosidade da mesma. ${ }^{20}$

\section{CONCLUSÃO}

O uso de energia de microondas para polimerização de resinas acrílicas é um excelente método. Por ser rápido e limpo, proporciona uma economia processual extremamente grande na confecção de bases de dentaduras.

As deficiências de propriedade causadas às resinas convencionais pela polimerização por microondas podem ser controladas com adaptação do método sem perda de suas vantagens.

Os estudos apontam que resinas próprias para microondas devem ser aperfeiçoadas para que possam ser utilizadas irrestritamente na construção de próteses totais, parciais removíveis e reembasamentos.

A polimerização de resinas por microondas pode constituir uma alternativa importante para a produção de próteses totais com maior agilidade e menor custo na rede pública de assistência à saúde. 


\section{REFERÊNCIAS}

1. ALMEIDA, M. H. W.; DOMITTI, S. S.; CONSANI, S.; CONSANI, R. L. X.; MILAN, F. M. Influência de técnicas de polimerização sobre a adaptação das bases de prótese total. Revista da Faculdade de Odontologia de Passo Fundo, Passo Fundo, v. 4, n. 1, p. 49-56, jan./jun. 1999.

2. NISHII, M. Studies on the curing of denture base resins with microwave irradiation: with particular reference to heat-curing resins. Journal of Osaka University Dental School, Osaka, v. 2, p. 23-40, 1968.

3. PHILLIPS, W. R. Química das resinas sintéticas. In: Skiner materiais dentários. 9 ed. Rio de Janeiro: Guanabara Koogan,1993. p. 91-102.

4. KIMURA, H. TERAOKA, F.; OHNISHI, H.; SAITO, T.; YATO, M. Application of microwave for dental technique (part 1). Journal of Osaka University Dental School, Osaka, v. 23, p. 43-49. 1983.

5. KIMURA, H.; TERAOKA, F.; SAITO, T. Application of microwave for dental technique (part 2). Journal of Osaka University Dental School, Osaka, v. 24, p. 21-29, 1984.

6. LEVIN, B.; SANDERS, J. L.; REITZ, P. V. The use of microwave energy for processing acrylic resins. Journal Prosthetic Dentistry, St. Louis, v. 61, n. 3, p. 381-383, Mar. 1989.

7. BARBOSA, C. M. R.; DEL BEL CURY, A. A.; PANZERI, H. Influencia da sorção de água e do processo de polimerização por energia de microondas na adaptabilidade das próteses totais. Revista de Odontologia da Universidade de São Paulo, São Paulo, v. 12, n. 2, p. 173-180, abr./jun. 1998.

8. BARONCINI NETO, Z. Estudo das Alterações de resinas acrílicas temopolimerizaveis processadas em forno de microondas convencional. Bauru, 1997. Dissertação (Mestrado em Odontologia) - Faculdade de Odontologia de Bauru, Universidade de São Paulo.

9. BARTOLONI, J. A.; MURCHISON, D. F.; WOFFORD, D. T.; SARKAR, N. K. Degree of conversion in denture base materials for varied polymerization techniques. Journal of Oral Rehabilitation, Birmingham, v. 27, n. 6, p. 488-493, June, 2000.

10. DEL BEL CURY, A. A.; RODRIGUES JUNIOR, A. L.; PANZERI, H. Resinas acrílicas dentais polimerizadas por energia de microondas, método convencional de banho de água e quimicamente ativada: propriedades físicas. Revista de Odontologia da Universidade de São Paulo, São Paulo, v. 8, n. 4, p. 243-249, out./dez. 1994.

11. GARCIA, R. C. M.; DEL BEL CURY, A. A. Reembasamento de bases de prótese total: método convencional e por microondas. Revista de Odontologia da Universidade de São Paulo, São Paulo, v. 10, n. 4, p. 295-302, out./ dez. 1996. 
12. SADAMORI, S.; ISHII, T.; HAMADA, T. Influence of thickness on the linear dimensional change, warpage, and water uptake of a denture base resin. International Journal of Prosthodontic. St. Louis, v. 10, n. 1, p. 35-43, 1997.

13. SANDERS, J.L.; LEVIN, B.; REITZP. V. Porosity in denture acrylic resin cured by microwave energy. Quintessence International, Berlin, v. 18, n. 7, p. 453-456, Nov. 1987.

14. BRAUN, K. O.; DEL BEL CURY, A. A.; CURY, J. A. Avaliação in vitro da efetividade de polimerização da resina acrílica através da energia de microondas, quando em contato com metal. Revista de Odontologia da Universidade de São Paulo, São Paulo, v. 9, n. 3, p. 197-206, jul./set. 1995.

15. AL DOORI, D.; HUGGETT, R.; BATES, J. F.; BROOKS, S. C. A comparison of denture base acrylic resins polymerized by microwave irradiation and by conventional water bath curing systems. Dental Materials, v. 4, n. 1, p. 25-32, Feb. 1988.

16. REITZ, P. V.; SANDERS, J. L.; LEVIN, B. The curing of denture acrylic resins by microwave energy. Physical properties. Quintessence International, Berlin, v. 6, n. 8, p. 547-541, Aug. 1985.

17. YANNIKAKIS, S.; ZISSIS, A.; POLYZOIS, G.; ANDREOPOULOS, A. Evaluation of porosity in microwave-processed acrylic resin using a photographic method. Journal of Prosthetic Dentistry, St. Louis, v. 87, n. 6, p. 615-619, June, 2002.

18. PHILLIPS, W. R. Resinas para base de dentadura: considerações técnicas e resinas diversas. In:

. Skiner materiais dentários. 9 ed. Rio de Janeiro: Guanabara Koogan,1993. p. 103-123.

19. BLAGOJEVIC, V.; MURPHY, V. M. Microwave polymerization of denture base materials. A comparative study. Journal of Oral Rehabilitation, Birmingham, v. 26, n. 10, p. 804-808, Oct. 1999.

20. BRAUN, K. O.; RODRIGUES GARCIA, R. C. M.; RIZZATTI-BARBOSA, C. M.; DEL BEL CURY, A. A. Alterações dimensionais lineares de resinas para base de próteses polimerizadas por microondas. Pesquisa Odontológica Brasileira, São Paulo, v. 14, n. 3, p. 278-282, jul./set. 2000.

21. MEMON, M. S.; YUNUS, N.; RAZAK, A. A. A. Some mechanical properties of a highly cross-linked, microwave-polymerized, injection-molded denture base polymer. International Journal of Prosthodontic, St. Louis, v. 14, n. 3, p. 214-218, 2001.

22.JAGGER, R. G.; HUGGET, R. The effect of cross-linking on sorption properties of denture-base material. Dental Materials, Washington, v. 6, n. 4, p. 276-278, Oct. 1990.

23. MAY, K. B.; SHOTWELL, J. R.; KORAN, A.; WANG, R. Color stability: denture base resins processed whit the microwave method. Journal of Prosthetic Dentistry, St. Louis, v. 76, n. 6, p. 581-589, Dec. 1996. 
24. SHERIDAN, P. J.; KOKA, S.; EWOLDSEN N. O.; LEFEBVRE, C. A.; LAVIN, M. T. Cytotoxicity of denture base resins. International Journal of Prosthodontic, St. Louis, v. 88, n. 2, p. 145-150, Aug. 2002.

25. JAGGER, R. G. Effect of the curing cycle on some properties of a polimethyl methacrylate denture base material. Journal of Oral Rehabilitation, Birmingham, v. 5, n. 2, p. 151-157, Apr. 1978.

26. DEL BEL CURY, A. A.; RACHED, R. N.; GANZAROLLI, S. M. Microwave-cured acrylic resins and silicone-gypsum moulding technique. Journal of Oral Rehabilitation, Birmingham, v. 28, n. 5, p. 433-438, May, 2001.

27. TERAOKA, F.; TAKAHASHI, J. Controlled polymerization system for fabricating precise dentures. Journal of Prosthetic Dentistry, St. Louis, v. 83, n. 5, p. 514520, May, 2000.

28. SCHNEIDER, R. L.; CURTIS, E. R.; CLANCY, J. M. S. Tensile bond strength of acrylic resin denture teeth to a microwave or heat-processed denture base. Journal of Prosthetic Dentistry, St. Louis, v. 87, n. 6, p. 615-619, June, 2002.

29. MONTEIRO NETTO, J.; MUENCH, A.; MIRANDA JUNIOR, W. G. Influência dos tipos de polimerização na resistência transversal da resina para base de prótese total. RPG Revista de Pós-Graduação. São Paulo, v. 6, n. 3, p. 245-248, jul./set. 1999.

30. SILVA, F. A. P.; SILVA, T. B. P.; DEL BEL CURY, A. A. Effect of intrinsic pigmentation on the flexural strength of a microwave-cured acrylic resin. Brazilian Dental Journal, São Paulo, v. 15, n. 3, p. 205-207, Mar, 2002.

31. TAKAMATA, T. SETCOS J. C.; PHILLIPS R. W.; BOONE M. E. Adaptation of acrylic resin dentures as influenced by the activation mode of polymerization. Journal of American Dental Association, Chicago, v. 119, n. 2, p. 271-276, Aug. 1989.

32. KIMURA, H.; TERAOKA, F.; SUGITA, M. Application of microwave for dental technique (part 3). Journal of Osaka University Dental School, Osaka, v. 27, p. 41-50, 1987.

33. SADAMORI, S.; SISWOMIHARDJO, W.; KAMEDA, K.; SAITO, A.; HAMADA, T. Dimensional changes of relined denture bases with heat-cured, microwave-activated, autopolymerizing, and visible light-cured resins. A laboratory study. Australian Dental Journal, St. Leonard, v. 40, n. 5, p. 322-326, 1995.

Endereço para correspondência:

R. Padre Agostinho, 2885, ap.1704 B3

CEP 80710-000 Bigorrilho - Curitiba - PR

Fones: (41) 3323-4897 / 8818-8725

savio@ufpr.br 
